\title{
Maintenance of Fitness and Quality-of-Life Benefits From Supervised Exercise Offered as Supportive Care for Breast Cancer
}

Amy A. Kirkham, PhDa; Kelcey A. Bland, MScb; Holly Wollmann, MScc; Alis Bonsignore, MScd; Don C. McKenzie, MD, PhDc; Cheri Van Patten, MSce; Karen A. Gelmon, MDe; and Kristin Campbell, PhD, BScPTc

\begin{abstract}
Background: Overwhelming randomized controlled trial evidence demonstrates that exercise has positive health impacts during and after treatment for breast cancer. Yet, evidence generated by studies in which exercise programs are delivered outside a tightly controlled randomized trial setting is limited. The purpose of this study was to assess the effectiveness of an evidence-based exercise program with real-world implementation on physical fitness and quality of life ( $\mathrm{QoL}$ ). Patients and Methods: Oncologists referred women with early-stage breast cancer who were scheduled to receive adjuvant chemotherapy. The program consisted of supervised aerobic and resistance exercise of moderate to vigorous intensity 3 times per week until the end of treatment (chemotherapy \pm radiotherapy), then twice per week for 10 weeks, followed by once per week for 10 weeks. Health-related physical fitness and QoL were assessed at baseline, end of treatment, end of program, and 1-year follow-up. Results: A total of 73 women were enrolled. Estimated peak $\mathrm{VO}_{2}\left(\mathrm{VO}_{2}\right.$ peak), $\mathrm{QoL}$, and body weight were maintained between baseline and end of treatment, whereas muscular strength improved $(P<.01)$. By the end of the program, $\mathrm{VO}_{2}$ peak, heart rate recovery, waist circumference, and some aspects of $\mathrm{Q}$ L were improved (all $P<.01$ ) relative to baseline. One year later, $\mathrm{VO}_{2}$ peak, $\mathrm{QoL}$, and waist circumference were maintained relative to end of program, whereas the improvements in strength and heart rate recovery had dissipated (all $P<.01$ ). Conclusions: Evidence-based exercise programming delivered with real-world implementation maintained $\mathrm{VO}_{2}$ peak, strength, and $\mathrm{CoL}$ during adjuvant treatment and improved these measures after treatment completion among women with breast cancer. Continued guidance and support may be required for long-term maintenance of strength improvements in this population.
\end{abstract}

J Natl Compr Canc Netw 2019;17(6):695-702 doi: $10.6004 /$ jnccn.2018.7276

a University of Alberta, Edmonton, Alberta, Canada; ${ }^{b}$ Australian Catholic University, Melbourne, Australia; 'University of British Columbia, Vancouver, British Columbia, Canada; ${ }^{d}$ University of Toronto, Toronto, Ontario, Canada; and ${ }^{~}$ British Columbia Cancer Agency, Vancouver, British Columbia, Canada.

\section{Background}

A critical aspect of breast cancer survivorship care includes addressing the negative sequelae of breast cancer therapy on health-related fitness and quality of life (QoL). The NCCN Clinical Practice Guidelines in Oncology (NCCN Guidelines) for Breast Cancer state that support for exercise, healthy eating, and weight management are important standards for survivorship care. ${ }^{1}$ However, within most cancer treatment centers, exercise programming is not offered and access to registered dietitians is limited.

Although exercise training can be beneficial during and after treatment, intervention during chemotherapy may be especially important as supportive care in managing treatment-related side effects. With respect to exercise during adjuvant chemotherapy for breast cancer, randomized controlled trials (RCTs) have demonstrated that supervised aerobic or resistance exercise training has superior effects to both usual care and home-based exercise on health-related physical fitness and QoL.,3 Furthermore, the large $(\mathrm{N}=300)$, multicenter, randomized CARE trial (ClinicalTrials.gov identifier: NCT00249015) demonstrated that a combined aerobic and resistance training intervention provided equivalent or greater benefits across these health domains relative to aerobic exercise alone. ${ }^{4}$ Synthesis of these major efficacy trial results suggests that the most effective format of exercise programming for women with breast cancer during adjuvant chemotherapy is supervised aerobic and resistance exercise training.

With efficacy established, a key element in developing an evidence-based approach for using exercise and healthy eating as supportive therapy during chemotherapy for breast cancer is translating these benefits into effective clinical programming. The effectiveness of an intervention is measured by its ability to produce a similar effect under real-world conditions. ${ }^{5}$ Defining features of an effectiveness trial include minimal exclusion criteria; flexible, individualized delivery of the intervention; and reduced expectations for adherence. ${ }^{5}$ Little is known about the effectiveness of supervised exercise training in patients with breast cancer when the program is 
implemented in a clinical setting outside the stringent conditions of an RCT. ${ }^{6}$

The NExT study (ClinicalTrials.gov identifier: NCT01806181) assessed the effectiveness of exercise and healthy eating programming offered to women receiving adjuvant chemotherapy for early-stage breast cancer. Exercise programming during treatment was closely modeled after the combined aerobic and resistance exercise arm of the CARE efficacy trial. ${ }^{4}$ The NExT exercise program is unique from programs used in previous studies by continuing for an additional 20 weeks after treatment completion, with a gradual step-down in supervised exercise. The program's reach, its implementation, and the effectiveness and maintenance of self-reported physical activity and the physical component summary of the RAND-36 have been reported previously. ${ }^{7}$ In brief, goals for successful reach were attained $(>50 \%$ referral rate, $>70 \%$ uptake, and retention) and aerobic and resistance physical activity and the physical component summary increased by the end of the program, but only aerobic physical activity and the physical component summary remained higher than baseline at 1-year follow-up.

The goal this study was to assess the changes in objective measures of health-related physical fitness (eg, aerobic fitness, muscular strength, body composition) and QoL throughout the program, and maintenance of these changes for 1 year after program completion. Based on previous findings derived from efficacy trials, it was hypothesized that the NExT program would attenuate negative treatment-related effects on aerobic fitness, strength, body weight, and QoL, and result in improvements in aerobic fitness, strength, body weight, and QoL after treatment completion. It was also hypothesized that the step-down approach would result in maintenance of improvements for 1 year after program completion.

\section{Patients and Methods}

\section{Study Design and Participants}

The NExT study was a single-arm intervention offered to English-speaking adult women with stages I-IIIA breast cancer who were scheduled to receive adjuvant chemotherapy at the Vancouver Center of the British Columbia Cancer Agency (BCCA). Participants were recruited via a "prescription" from a medical oncologist. Participants had to be able to complete the baseline assessment before completion of $>50 \%$ of chemotherapy treatment and be deemed safe to exercise by their oncologist. Patients with a body mass index $>40 \mathrm{~kg} / \mathrm{m}^{2}$, uncontrolled cardiovascular disease or diabetes, or disabilities impeding exercise were excluded. The 12 -month recruitment period originally planned as a measure of yearly intake for a potential clinical program was extended to 15 months after securement of additional funding. The BCCA Research Ethics Board approved this study and participants provided informed consent.

\section{Intervention}

Participants were invited to take part in the exercise training program and a single nutrition information session. The exercise program had 3 distinct phases: (1) treatment (baseline/enrollment to completion of chemotherapy \pm radiotherapy, 3 times/wk); (2) posttreatment (10 weeks, twice/wk); and (3) maintenance (10 weeks, once/wk) (Figure 1). The exercise program has been described previously in detail. ${ }^{8}$ In brief, supervised aerobic and whole-body resistance sessions were supplemented with encouragement of home-based exercise to work toward achieving the NCCN recommendation of 150 minutes of moderate-intensity aerobic activity and 2 or 3 sessions of whole-body resistance training per week (Figure 1). ${ }^{1}$ The single, 2 -hour, small group-based nutrition session involved discussion of topics ranging from nutritional management of treatment side effects, achieving adherence to Canada's Food Guide and Canadian Cancer Society guidelines, and Dietary Reference Intake.

\section{Outcome Measures}

Demographics were collected via questionnaire. Treatment and diagnosis characteristics were extracted from

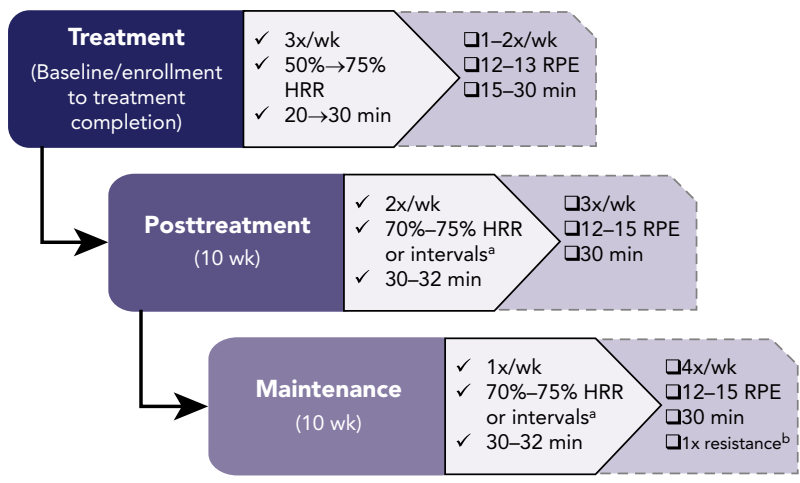

Figure 1. NExT exercise prescription for each of the 3 phases of exercise training program. Check marks indicate supervised aerobic session prescription; supervised whole-body resistance sessions were performed at the same sessions. Open boxes indicate home-based session of aerobic type unless otherwise specified; a log book provided the prescriptions, including the RPE scale to measure intensity of exercise, and a place to record exercise performed. Arrows indicate progression.

Abbreviations: HRR, heart rate reserve; RPE, Rating of Perceived Exertion.

${ }^{a}$ Aerobic intervals ( 4 times: 4 minutes hard, 4 minutes easy ${ }^{8}$ ) were prescribed for participants without a history of cardiovascular disease, current heart medications, dyspnea/asthma issues, or injuries limiting exercise intensity. bParticipants could perform the supervised program at their own gym according to the intervention prescription or were provided with a resistance band program. 
medical records. Assessments included physical fitness tests and questionnaires, and were performed at the following time points: (1) baseline/enrollment, (2) end of treatment (1 week after radiotherapy if received, or the length of one chemotherapy cycle after the last cycle), (3) end of program (completion of intervention, 20 weeks after end of treatment), and (4) 1-year follow-up (1 year after end of program without further intervention).

Health-related physical fitness was assessed using methods that were deemed to be practical and costeffective for a clinical program. Because of the focus on practicality, familiarization sessions were not performed. These assessments were used to individualize exercise prescriptions and to evaluate progress and program effectiveness. Waist circumference was measured as the average of 2 measurements $\leq 0.5 \mathrm{~cm}$ apart around the top of the iliac crest. ${ }^{9}$ Aerobic fitness was estimated using a modified Balke treadmill test that was terminated at $70 \%$ of age-predicted heart rate reserve. ${ }^{10}$ When the target heart rate was reached, the speed and grade were immediately returned to $2.0 \mathrm{mph}$ and $0 \%$, respectively, for an active recovery. Heart rate was recorded at the end of each 3-minute stage of the test and at 2 minutes into recovery. Participants were asked to minimize talking and handrail holding during the test and recovery period. For each test, a linear regression of the heart rate at the end of each stage and the corresponding $\mathrm{VO}_{2}$ was performed and solved for age-predicted peak heart rate $(206-[0.88 \times \text { age }])^{11}$ to estimate peak $\mathrm{VO}_{2}$ $\left(\mathrm{VO}_{2}\right.$ peak). $\mathrm{VO}_{2}$ was calculated for each workload of the test using a metabolic equation, ${ }^{12}$ which we have previously shown to be accurate in this population. ${ }^{13}$ Heart rate recovery, a known correlate of $\mathrm{VO}_{2}$ peak in patients with breast cancer, ${ }^{14}$ was calculated as the peak heart rate during the test minus the heart rate at 2 minutes into recovery. One-repetition maximums (1-RMs) for chest press and leg press were estimated from validated prediction equations using maximal weight lifted for 7 to 10 repetitions as previously used in efficacy trials, such as the CARE trial. ${ }^{4,15}$ As an indicator of feasibility of our fitness assessment methods, we tracked the number of failures and reasons for failure of completion of assessments.

General and breast cancer-specific health-related QoL was assessed by the RAND-36 ${ }^{16}$ and the Functional Assessment of Cancer Therapy-Breast (FACT-B), respectively. ${ }^{17}$ The questionnaire scales that relate most to physical components of QoL are reported because they are most likely to respond to interventions that change physical morbidity. ${ }^{18}$ QoL was also assessed at the completion of chemotherapy to compare the change scores across chemotherapy reported in the CARE efficacy trial as the primary and secondary outcomes.
The differences between means for each measure were compared with the established minimally important differences (RAND-36 $=3$; FACT-B trial outcome index $=5$; FACT-General $=3)^{19,20}$

\section{Statistics}

Each health-related physical fitness and QoL outcome was analyzed using a generalized linear mixed model in SPSS Statistics, version 24 (IBM Corporation, Armonk, NY), which does not require that participants with missing data be dropped from the analysis. All available data, including for participants who dropped out of the intervention, were included. A random intercept was included to account for intraindividual similarities across time points, and time point was included as a repeated and fixed effect. Significant time effects were interpreted with post hoc contrasts for each of the 3 follow-up time points relative to baseline, end of treatment relative to end of program, and end of program relative to 1-year follow-up. Differences between time points were considered significant when $P \leq .01$, using the Bonferroni adjustment for multiple comparisons. To compare variables in common between the NExT and CARE trials, change scores were calculated for QoL at the end of chemotherapy and for physical fitness at the end of treatment to match the format of data reported in CARE.

\section{Results}

\section{Participants}

Between August 2013 and October 2014, a total of 109 patients were referred; 93 were eligible and 73 enrolled (78\% of eligible) (flow diagram previously published ${ }^{7}$ ). Table 1 describes the baseline characteristics of study participants; 5 participants withdrew before completion of any exercise sessions, and another 4 withdrew during chemotherapy. Of these 9 withdrawals, 4 returned to complete outcome measures during the intervention period but not at 1-year follow-up. Among participants who did not withdraw, $83 \%$ completed 1-year follow-up assessments.

The treatment phase duration was $25 \pm 8$ weeks and the entire program was $45 \pm 8$ weeks, on average. Average attendance during treatment, posttreatment, and maintenance phases were $60 \% \pm 26 \%, 52 \% \pm 33 \%$, and $50 \% \pm 38 \%$, respectively.

\section{Body Weight and Waist Circumference}

Body weight did not change over time $(P=.11)$ (Table 2). Waist circumference did not change between baseline and end of treatment $(P=.03)$ or between end of treatment and end of program $(P=.02)$, but it was significantly lower at end of program and at 1-year 


\begin{tabular}{|c|c|c|}
\hline Characteristic & $\mathbf{n}$ & $\%$ \\
\hline \multicolumn{3}{|l|}{ Demographics } \\
\hline Age, mean $\pm S D$ (range), y & \multicolumn{2}{|c|}{$50.8 \pm 10.6(29-77)$} \\
\hline \multicolumn{3}{|l|}{ Marital status ${ }^{\mathrm{a}}$} \\
\hline Married/Common-law & 49 & 71 \\
\hline Divorced/Separated & 5 & 7 \\
\hline Single & 11 & 16 \\
\hline Widowed & 3 & 4 \\
\hline \multicolumn{3}{|l|}{ Ethnicitya } \\
\hline White & 44 & 64 \\
\hline Asian & 22 & 32 \\
\hline South Asian & 2 & 3 \\
\hline Black or African American & 1 & 1 \\
\hline \multicolumn{3}{|l|}{ Education $^{a}$} \\
\hline High school diploma & 5 & 7 \\
\hline Technical/Community college & 18 & 26 \\
\hline Some university & 8 & 12 \\
\hline Bachelor's degree & 17 & 25 \\
\hline Higher than bachelor's degree & 21 & 30 \\
\hline \multicolumn{3}{|l|}{ Work status during treatment } \\
\hline Working during & 11 & 15 \\
\hline Not working & 55 & 75 \\
\hline Part-time/Casual & 7 & 10 \\
\hline \multicolumn{3}{|l|}{ Medical history } \\
\hline \multicolumn{3}{|l|}{ Menopausal status } \\
\hline Postmenopausal (natural) & 27 & 37 \\
\hline Surgery/Hysterectomy & 6 & 8 \\
\hline Perimenopausal & 12 & 16 \\
\hline Menopausal & 28 & 38 \\
\hline \multicolumn{3}{|l|}{ Comorbidities } \\
\hline Hypertension & 12 & 16 \\
\hline Mental illness & 11 & 15 \\
\hline Arthritis/Osteoporosis & 8 & 11 \\
\hline Asthma/Lung disease & 7 & 10 \\
\hline Cardiovascular disease/arrhythmias & 5 & 7 \\
\hline Metabolic disease & 3 & 4 \\
\hline Previous cancer & 3 & 4 \\
\hline Thyroid disorder & 3 & 4 \\
\hline Fibromyalgia/Multiple sclerosis & 2 & 3 \\
\hline
\end{tabular}

(continued) aMissing 4 responses from participants who withdrew before completing demographics questionnaire.

follow-up relative to baseline $(P<.01)$. Waist circumference did not change between end of program and 1-year follow-up $(P=.86)$.

\begin{tabular}{|c|c|c|}
\hline Characteristic & $\mathbf{n}$ & $\%$ \\
\hline \multicolumn{3}{|l|}{ Cancer diagnosis and treatment } \\
\hline \multicolumn{3}{|l|}{ Tumor stage } \\
\hline I & 17 & 23 \\
\hline II & 47 & 64 \\
\hline III & 9 & 12 \\
\hline \multicolumn{3}{|l|}{ Chemotherapy regimen } \\
\hline Anthracycline-containing & 51 & 70 \\
\hline Taxane-containing & 70 & 96 \\
\hline Cyclophosphamide-containing & 73 & 100 \\
\hline \multicolumn{3}{|l|}{ Primary surgery } \\
\hline Breast-conserving surgery & 53 & 73 \\
\hline Mastectomy & 20 & 27 \\
\hline Radiotherapy & 67 & 92 \\
\hline \multicolumn{3}{|l|}{ Hormone therapy } \\
\hline Aromatase inhibitor & 16 & 22 \\
\hline Selective estrogen receptor modulator & 44 & 60 \\
\hline None & 13 & 18 \\
\hline
\end{tabular}

aMissing 4 responses from participants who withdrew before completing demographics questionnaire.

\section{Aerobic Fitness}

Estimated $\mathrm{VO}_{2}$ peak could not be calculated in 16 of 249 (6\%) completed assessments due to $\beta$-blocker use $(n=11)$, heart rate monitor failures $(n=3)$, and failure to complete stage 2 before achieving target heart rate $(n=2)$. No significant change was seen in $\mathrm{VO}_{2}$ peak from baseline to end of treatment $(P=.34)$ or from end of treatment to end of program $(P=.02)$ (Table 2$). \mathrm{VO}_{2}$ peak was significantly higher at end of program relative to baseline $(P<.01)$. At 1-year follow-up, $\mathrm{VO}_{2}$ peak was not different from baseline $(P=.13)$ or end of study $(P=.06) . \mathrm{VO}_{2}$ peak baseline values and percentage change during treatment were similar between the NExT and CARE trials (Figure 2A, C).

Relative to baseline, heart rate recovery did not change by end of treatment $(P=.19)$, but it was improved by end of program relative to both end of treatment and baseline (both $P<.01$ ) (Table 2). However, this improvement was transient, because at 1-year follow-up, it was not different from baseline $(P=.45)$ and was significantly lower than at end of program $(P<.01)$.

\section{Muscular Strength}

The chest press test was not completed in 36 of 249 assessments (14\%) due to breast surgery-related limitations $(n=25)$, non-cancer-related injury $(n=10)$, and time limitations $(n=1)$. The leg press test was not completed in 10 assessments (4\%) due to non-cancer-related 


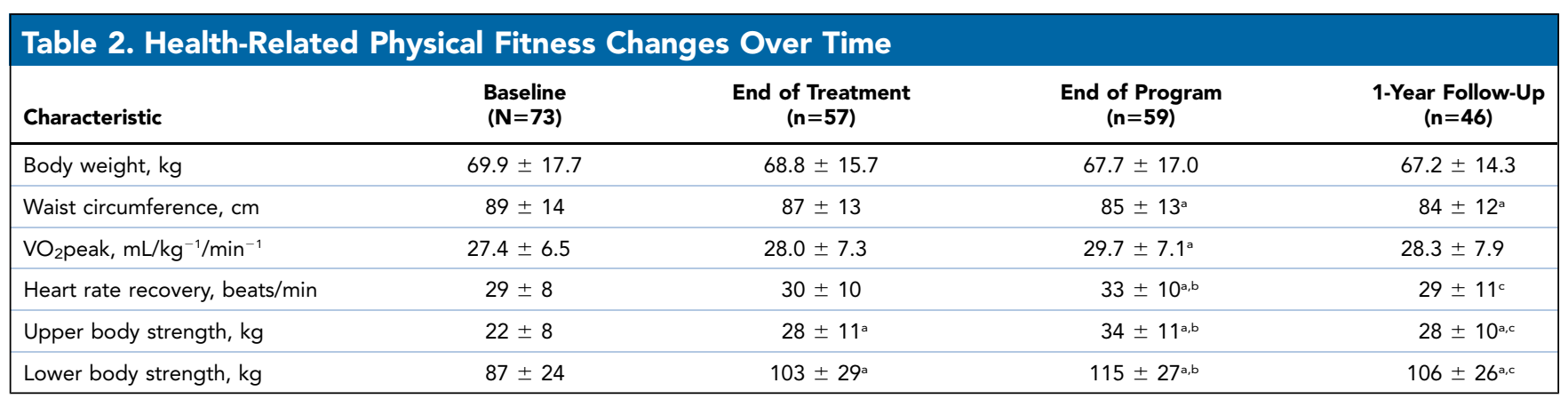

aSignificantly different from baseline with $P \leq .01$.

bSignificantly different from end of treatment with $P \leq 01$.

cSignificantly different from end of program with $P \leq .01$.

injury $(\mathrm{n}=9)$ and time limitations $(\mathrm{n}=1)$. The pattern of change for estimated 1-RM was the same for chest press and leg press, with end of treatment, end of program, and 1-year follow-up significantly improved from baseline (all $P<.01$ ) (Table 2). An additional significant improvement between end of treatment and end of program (both $P<.01$ ) was seen, but a significant decrease was observed from end of program to 1-year follow-up (both $P<.01$ ). Baseline chest press and leg press 1-RMs were similar between the NExT and CARE trials, whereas percent change during treatment was substantially larger for NExT (Figure 2C).

\section{Quality of Life}

Between baseline and end of treatment, no QoL measures changed (all $P>.06$ ). Between end of treatment and end of program, the RAND-36 physical functioning, role limitations due to physical health, energy/fatigue, and general health scales and FACT-General scores were improved (all $P<.01$ ) (Figure 3). These improvements in the RAND-36 role limitations due to physical health and the FACT-General score at end of program were significant relative to baseline (both $P<.01$ ). At 1-year follow-up, all scores were higher than baseline (all $P<.01$ ), except for general health $(P=.15)$. Relative to end of program, only the FACT-B trial outcome index was improved $(P<.01)$ at 1-year follow-up. Among the baseline values of QoL measures in common between the NExT and CARE trials, role limitations due to physical health was lower in NExT, whereas all other measures were similar (Figure 2B). The percentage change scores for QoL during chemotherapy were larger for NExT (using the end-of-treatment time point) than for the CARE trial for physical functioning, role limitations due to physical health, and the physical component summary (Figure 2D).

\section{Discussion}

Although numerous randomized trials have demonstrated the efficacy of exercise training in generating positive effects on health-related physical fitness and QoL in women with breast cancer, the NExT trial contributes new information regarding the effectiveness of exercise training when delivered in a setting more closely resembling a real-world model of a clinical program. Expected changes in the absence of intervention during treatment include a reduction in aerobic fitness, muscular strength, and QoL, and a gain in body weight. In line with our hypothesis, participants in the program experienced meaningful health benefits during adjuvant treatment, including maintenance of aerobic fitness, QoL, and body weight and improvement of upper and lower body muscular strength.

To allow for direct comparison of results with those of the CARE efficacy trial, ${ }^{4}$ the same QoL and fitness assessments were used at similar time points in NExT. Differences with the NExT trial included use of a submaximal treadmill test to estimate rather than measure $\mathrm{VO}_{2}$ peak and the performance of fitness assessments after the completion of all primary treatments (chemotherapy \pm radiotherapy) due to feasibility and staffing limitations. Participant demographics and diagnoses were comparable between the trials, but the chemotherapy regimens differed. It is unknown how different regimens might affect fitness and QoL outcomes. At baseline, QoL, $\mathrm{VO}_{2}$ peak, and strength were closely matched between the studies (Figure 2A, B). As expected for a primary defining feature of an effectiveness trial, ${ }^{5}$ exercise adherence was lower in NExT $(60 \% \pm 26 \%$ attendance) than in the CARE trial and other similar RCTs (70\%-83\% attendance). ${ }^{2-4,21-24}$ In comparing the QoL change scores in the 2 studies over the same time period (duration of chemotherapy only), the average deterioration in physical functioning, role limitations due to physical health, and the physical component summary were smaller, albeit with greater variability, in NExT. For fitness changes, upper and lower body strength increased in both studies during treatment, but a greater average change was seen after an average of 25 weeks of intervention in NExT compared with an average of 16 weeks in CARE. In terms of statistical changes within 
A

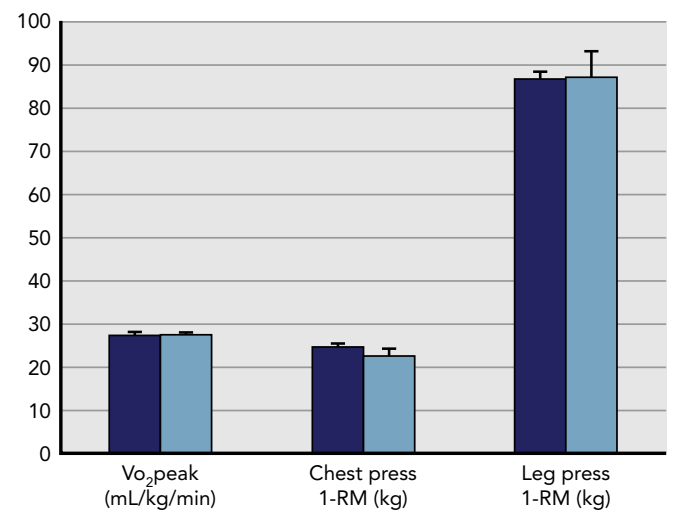

C

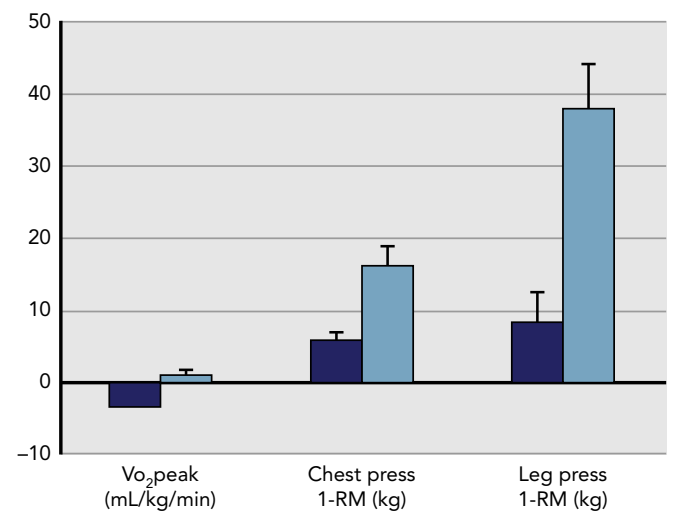

B

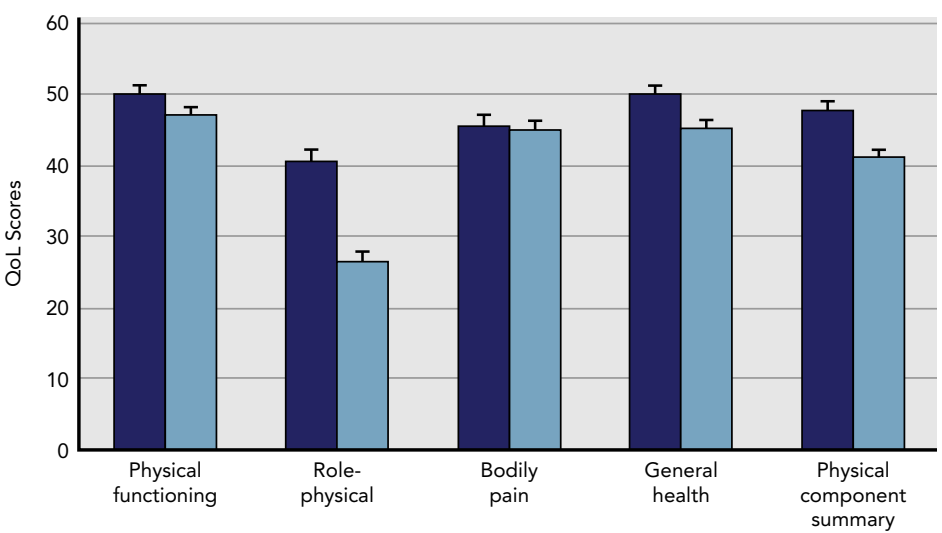

D

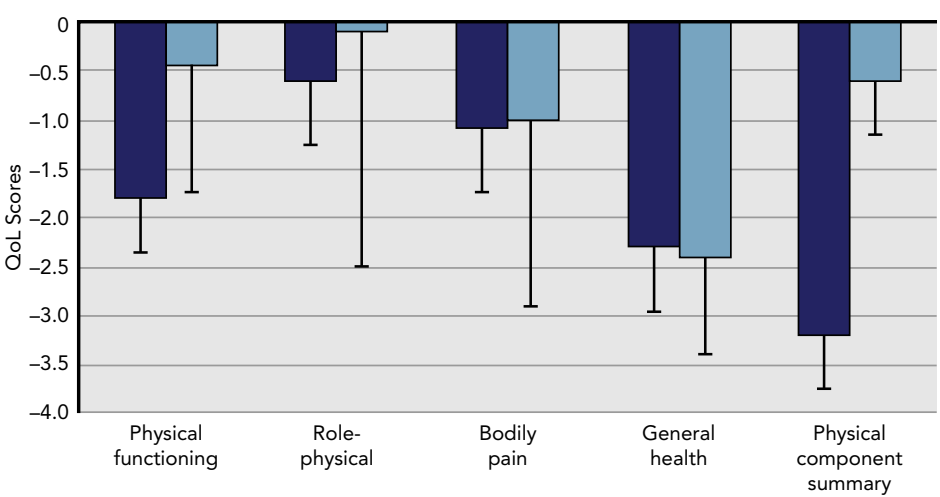

CARE

NExT

Figure 2. Comparison of (A) baseline fitness, (B) baseline $\mathrm{QoL}$, (C) percentage change in fitness, and (D) percentage change in QoL for the CARE efficacy trial's combined aerobic and resistance exercise arm and the NExT effectiveness trial's single exercise arm that was modeled after CARE. Data are mean \pm SE. Note that although change in QoL is assessed with similar timing in the 2 studies (start and end of chemotherapy), in the NExT trial, fitness was assessed only after completion of both chemotherapy and radiotherapy (and a longer exercise intervention), due to feasibility and staffing limitations, as opposed to after chemotherapy only in the CARE trial.

Abbreviations: 1-RM, one-repetition maximum; QoL, quality of life.

Data from Courneya KS, McKenzie DC, Mackey JR, et al. Effects of exercise dose and type during breast cancer chemotherapy: multicenter randomized trial. J Nat Cancer Inst 2013;105:1821-1832

each study, in the CARE trial the intervention only partially attenuated the treatment-related deterioration in $\mathrm{VO}_{2}$ peak and QoL; significant reductions still occurred. In NExT, changes in aerobic fitness, strength, body weight, and QoL were mitigated during treatment. A possible reason for the greater change in NExT is that participants were also encouraged to perform home-based aerobic exercise during treatment twice per week.

Regarding body composition, the CARE trial reported a body weight gain that was primarily due to increased lean mass as measured by dual-energy x-ray absorptiometry. ${ }^{4}$ We did not use advanced body composition assessment methods in NExT, but the lack of change in body weight combined with the nearly significant decrease in waist circumference may suggest findings similar to those of the CARE trial.
This study was also unique in that the total duration of exercise programming was considerably longer, with supervised sessions offered across the trajectory of breast cancer treatment. ${ }^{25}$ Despite reduced frequency of supervised sessions between the end-of-treatment and the end-of-program time points, there were improvements in strength, heart rate recovery, and QoL. Although we hypothesized, based on previous results of exercise efficacy trials, ${ }^{25}$ that aerobic fitness and body composition would improve with exercise training performed in the period after treatment completion, we saw only a trend toward improvement in $\mathrm{VO}_{2}$ peak and waist circumference between end of treatment and end of program.

Evidence is limited and inconsistent regarding maintenance of improvements in fitness and QoL after 


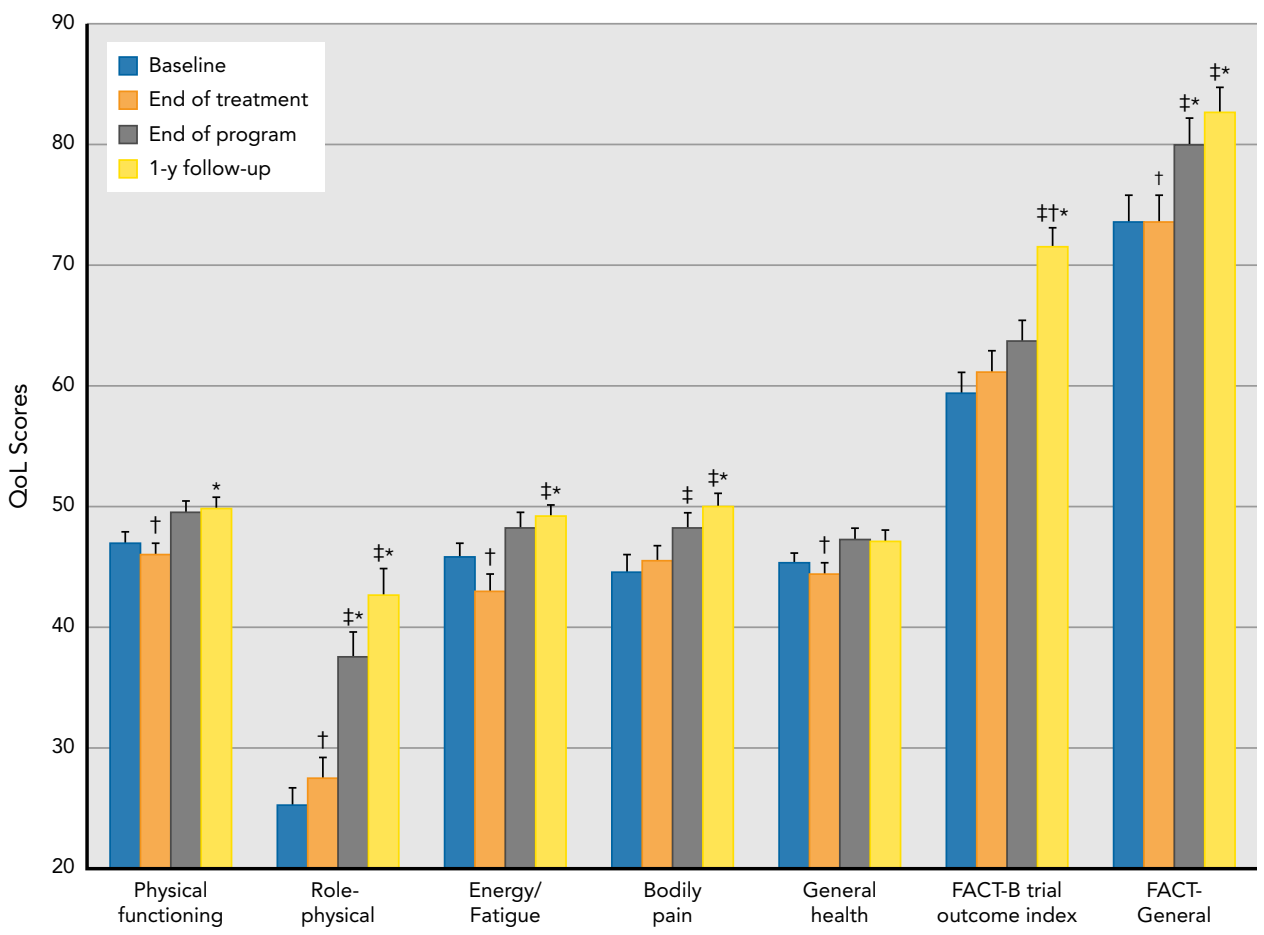

Figure 3. Health-related QoL changes for RAND-36 norm-based scores and FACT-General and FACT-B scores. Data are estimated marginal mean $\pm \mathrm{SE}$.

Abbreviations: FACT, Functional Assessment of Cancer Therapy; FACT-B, Functional Assessment of Cancer Therapy-Breast; QoL, quality of life.

*Significantly different from baseline.

†Significantly different from end of program.

¥Difference from baseline mean exceeds minimally important difference.

completion of a supervised exercise intervention during treatment of breast cancer. ${ }^{26}$ Three studies have reported that exercise effects on aerobic fitness, muscular strength, and QoL are not maintained without continued intervention. . $23,27^{2}$ One study reported maintenance of positive changes in QoL and aerobic fitness at 6 months after intervention, ${ }^{28}$ but dissipation of these benefits at 18 and 60 months was reported. ${ }^{29}$ Given the substantially longer duration of the NExT intervention, along with the step-down in supervised exercise frequency and increase in prescribed home-based exercise, we hypothesized that benefits would be maintained for 1 year. The objectively measured changes in aerobic fitness and muscular strength reported in the present study mirror changes in self-reported exercise habits previously reported for the NExT program. ${ }^{7}$ Aerobic and resistance exercise levels were higher at end of program relative to baseline, but at 1-year follow-up, only the aerobic levels remained higher than baseline and unchanged relative to end of program. ${ }^{7}$ This suggests that there may be more barriers to engaging in resistance training (eg, access to equipment, less familiarity) and that more guidance and support, including behavior modification strategies (eg, goal setting, self-monitoring), are needed to promote maintenance of muscular strength outside a supervised exercise program. However, an important finding for long-term health and functioning is that muscular strength at 1-year follow-up was still higher than at baseline.

The primary strength of our study design is the resemblance to real-world conditions with respect to the expectations regarding exercise adherence and the generalizability of the study population. Our study sample was much broader than in most prior RCTs with respect to age, ethnicity (one-third nonwhite), comorbid conditions, and work status during treatment (one-fourth worked at least part-time during treatment). A similar program could be implemented in clinical practice with minimal personnel cost (we estimated $\sim \$ 41,000 \mathrm{USD} / \mathrm{y}$ ) and use of clinical space and the purchase or donation of used exercise equipment. ${ }^{7}$

This study is not without limitations. There was no usual care control group for comparison. The singlearm design was chosen because it would more closely mimic operations of a real-world clinical program and thereby would attract a more similar population. Our use of estimated $\mathrm{VO}_{2}$ peak from a submaximal exercise test without gas analysis and the lack of familiarization testing sessions are limitations for interpretation of the fitness changes and for comparison with 
efficacy trials using these methods. These test formats were chosen to increase translatability to a real-world clinical program. The exercise component of the intervention was disproportionate relative to the nutrition component. For future studies and clinical programs, we recommend more ongoing nutritional education and feedback on dietary habits from a dietitian.

\section{Conclusions}

When delivered in real-world conditions, an exercise intervention based on synthesis of evidence from efficacy trials resulted in meaningful health benefits, including mitigation of the commonly observed treatmentrelated deterioration in aerobic fitness and QoL, improvement of muscular strength, and maintenance of body weight. We have also shown that a step-down approach to supervised exercise frequency after completion of primary adjuvant treatment seems to be beneficial for inducing lasting change for up to 1 year.

Submitted June 16, 2018; accepted for publication January 17, 2019.

Author contributions: Study concept: Kirkham, McKenzie, Van Patten, Gelmon, Campbell. Acquired funding: Van Patten, Campbell. Provided patients: Gelmon. Data acquisition: Kirkham, Bland, Wollmann, Bonsignore. Data analysis and interpretation: Kirkham. Manuscript preparation: Kirkham, Campbell.

Disclosures: The authors have not received any financial consideration from any person or organization to support the preparation, analysis, results, or discussion of this article.

Funding: This project was funded by the British Columbia Cancer Foundation. Dr. Kirkham was funded by the Canadian Institutes of Health Research.

Correspondence: Kristin Campbell, PhD, BScPT, University of British Columbia, 212-2177 Wesbrook Mall, Vancouver, BC, Canada V6T1Z3. Email: kristin.campbell@ubc.ca

\section{References}

1. Denlinger CS, Sanft T, Scott Baker K, et al. NCCN Clinical Practice Guidelines in Oncology: Survivorship. Version 2.2018. Accessed November 4, 2018. To view the most recent version, visit NCCN.org.

2. van Waart $H$, Stuiver MM, van Harten $W H$, et al. Effect of low-intensity physical activity and moderate- to high-intensity physical exercise during adjuvant chemotherapy on physical fitness, fatigue, and chemotherapy completion rates: results of the PACES randomized clinical trial. J Clin Oncol 2015;33:1918-1927.

3. Courneya KS, Segal RJ, Mackey JR, et al. Effects of aerobic and resistance exercise in breast cancer patients receiving adjuvant chemotherapy: a multicenter randomized controlled trial. J Clin Oncol 2007;25:4396-4404.

4. Courneya KS, McKenzie DC, Mackey JR, et al. Effects of exercise dose and type during breast cancer chemotherapy: multicenter randomized trial. J Natl Cancer Inst 2013;105:1821-1832.

5. Courneya KS. Efficacy, effectiveness, and behavior change trials in exercise research. Int J Behav Nutr Phys Act 2010;7:81.

6. Courneya KS, Rogers LQ, Campbell KL, et al. Top 10 research questions related to physical activity and cancer survivorship. Res Q Exerc Sport 2015;86:107-116

7. Kirkham AA, Van Patten CL, Gelmon KA, et al. Effectiveness of oncologistreferred exercise and healthy eating programming as a part of supportive adjuvant care for early breast cancer. Oncologist 2018;23:105-115.

8. Kirkham AA, Bonsignore A, Bland KA, et al. Exercise prescription and adherence for breast cancer: one size does not FITT all. Med Sci Sports Exerc 2018;50:177-186.

9. US Department of Health and Human Services, Public Health Service, National Institutes of Health, National Heart, Lung, and Blood Institute. The Practical Guide: Identification, Evaluation, and Treatment of Overweight and Obesity in Adults. Bethesda, MD; National Institutes of Health October 2000. NIH publication 00-4084.

10. Pollock ML, Foster C, Schmidt D, et al. Comparative analysis of physiologic responses to three different maximal graded exercise test protocols in healthy women. Am Heart J 1982;103:363-373.

11. Gulati M, Shaw LJ, Thisted RA, et al. Heart rate response to exercise stress testing in asymptomatic women: the St. James Women Take Heart Project. Circulation 2010;122:130-137.

12. Pescatello LS. ACSM's Guidelines for Exercise Testing and Prescription, 9th ed. Philadelphia, PA: Wolters Kluwer/Lippincott Williams \& Wilkins Health; 2014

13. Kirkham AA, Campbell KL, McKenzie DC. Comparison of aerobic exercise intensity prescription methods in breast cancer. Med Sci Sports Exerc 2013;45:1443-1450

14. Giallauria F, Vitelli A, Maresca L, et al. Exercise training improves cardiopulmonary and endothelial function in women with breast cancer: findings from the Diana-5 dietary intervention study. Intern Emerg Med 2016;11:183-189.
15. Knutzen KM, Brilla LR, Caine D. Validity of 1 RM prediction equations for older adults. J Strength Cond Res 1999;13:242-246.

16. Ware JE Jr. SF-36 Health Survey update. Spine 2000;25:3130-3139.

17. Brady MJ, Cella DF, Mo F, et al. Reliability and validity of the Functional Assessment of Cancer Therapy-Breast quality-of-life instrument. J Clin Oncol 1997;15:974-986.

18. Hemingway $H$, Stafford M, Stansfeld S, et al. Is the SF-36 a valid measure of change in population health? Results from the Whitehall II Study. BMJ 1997; 315:1273-1279.

19. Hays RD, Sherbourne CD, Mazel RM. The RAND 36-Item Health Survey 1.0. Health Econ 1993;2:217-227.

20. Webster K, Cella D, Yost K. The Functional Assessment of Chronic Illness Therapy (FACIT) measurement system: properties, applications, and interpretation. Health Qual Life Outcomes 2003;1:79.

21. Campbell A, Mutrie N, White F, et al. A pilot study of a supervised group exercise programme as a rehabilitation treatment for women with breast cancer receiving adjuvant treatment. Eur J Oncol Nurs 2005;9:56-63.

22. Kim CJ, Kang DH, Smith BA, et al. Cardiopulmonary responses and adherence to exercise in women newly diagnosed with breast cancer undergoing adjuvant therapy. Cancer Nurs 2006;29:156-165.

23. Travier N, Velthuis MJ, Steins Bisschop CN, et al. Effects of an 18-week exercise programme started early during breast cancer treatment: a randomised controlled trial. BMC Med 2015;13:121.

24. Segal R, Evans W, Johnson D, et al. Structured exercise improves physica functioning in women with stages I and II breast cancer: results of a randomized controlled trial. J Clin Oncol 2001;19:657-665.

25. Battaglini $C L$, Mills RC, Phillips BL, et al. Twenty-five years of research on the effects of exercise training in breast cancer survivors: a systematic review of the literature. World J Clin Oncol 2014;5:177-190.

26. Spark LC, Reeves MM, Fjeldsoe BS, et al. Physical activity and/or dietary interventions in breast cancer survivors: a systematic review of the maintenance of outcomes. J Cancer Surviv 2013;7:74-82.

27. Courneya KS, Segal RJ, Gelmon K, et al. Six-month follow-up of patientrated outcomes in a randomized controlled trial of exercise training during breast cancer chemotherapy. Cancer Epidemiol Biomarkers Prev 2007;16: 2572-2578.

28. Mutrie N, Campbell AM, Whyte F, et al. Benefits of supervised group exercise programme for women being treated for early stage breast cancer: pragmatic randomised controlled trial. BMJ 2007; 334:517

29. Mutrie N, Campbell A, Barry S, et al. Five-year follow-up of participants in a randomised controlled trial showing benefits from exercise for breast cancer survivors during adjuvant treatment: are there lasting effects? J Cancer Surviv 2012;6:420-430. 